\title{
Beam Based Alignment of the SLC Final Focus Superconducting Final Triplets*
}

\author{
P.RAIMONDI, P.J.EMMA, N.TOGE, N.J.WALKER and V.ZIEMANN \\ Stanford Linear Accelerator Center \\ Stanford University, Stanford CA 94305
}

\section{Abstract}

The strong demagnification inherent in final focus systems requires very high gradients for the final quadrupoles. Alignment of these magnets is critical in order to minimize the dispersion at the interaction point and backgrounds in the detector due to synchrotron radiation. Reported here is a technique for aligning the final quadrupoles with respect to the beam centroid, which makes use of measurements of the beam position downstream of the quadrupoles when their fields are varied. Results for the SLC final focus final superconducting triplets are presented, where a resolution of $<20 \mu \mathrm{m}$ is achieved.

\section{DESCRIPTION OF THE TECHNIQUE}

The basic idea to perform the alignment is that the beam trajectory is steered by a misaligned quadrupole in proportion to the quad strength and to the misalignment itself. The developed procedure can be summarized in four different steps:

1) Establish the reference orbit to which the quadrupoles are to be aligned.

2) Measure the BPM scale-factors variing the beam orbit using beam "bumps", with all the magnetic elements in the section off.

3) Evaluate the misalignment by changing the quadrupole field-strength and recording the orbit distortions.

4) Apply alignment correction with magnet movers and repeat step (3).

The first step is performed by turning off the triplets and centering the beam in the BPMs in the IP region using correctors upstream of the triplets (fig.1).

In the second step the BPM scale-factors are evaluated considering that between the correctors used to generate the bumps and the BPMs there are only drift spaces. In fig. 2 is shown typical behavior of the BPM readings when we apply bumps of different amplitudes. Particularly the orbit between

\section{MASTER}

*. Work supported by the Department of Energy, contract DE-ACO3-76SFO0515 the [NBP06,SBP06] should be a straight line, it is therefore very easy to extract the scale factors.

This "calibration" is crucial to avoid relative and systematic errors in the evaluation of the misalignments.

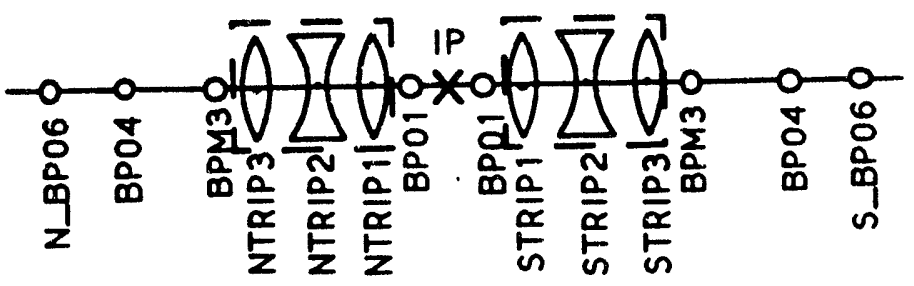

Figure 1. Schematic layout of the beam line in the IP region.

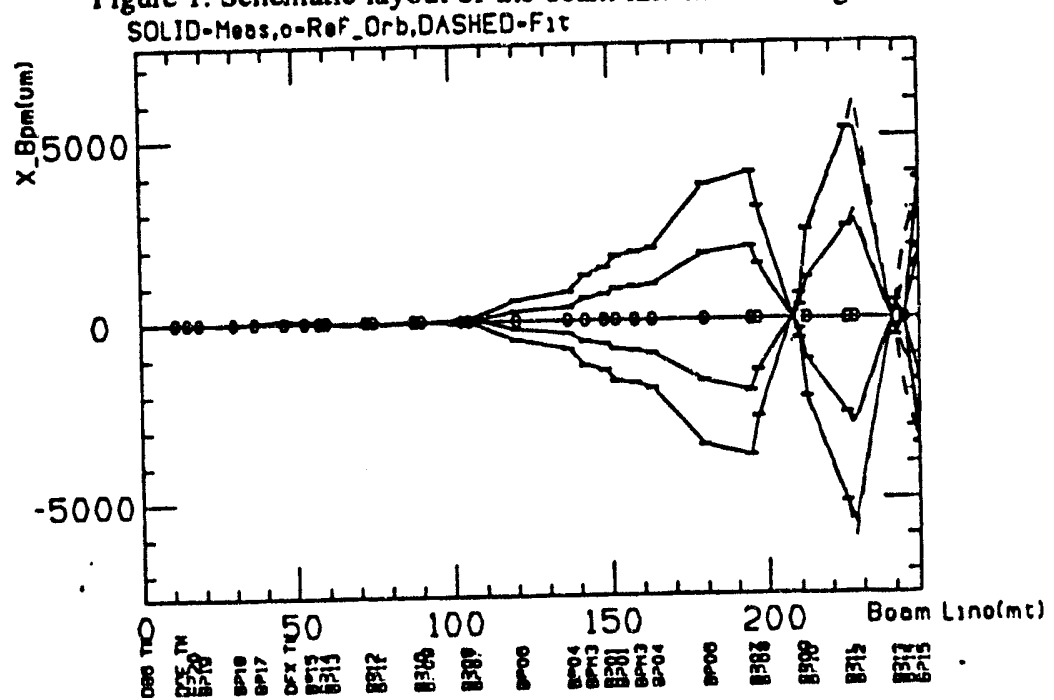

Figure 2. Beam orbit at different corrector values in the IP region as read from the BPMs with all the magnetic elements in the region [NBP06,SBP06] turned off.

Successively the triplet field is varied and the relative orbit distortions are measured using BPMs upstream and downstream of the triplets. As a further complication, it is not possible to act on the single quads independenty, however it can be shown that the beam centroid variation " $x$ " downstream of three quads Q1, Q2 and Q3 excited at a field strength " $q$ " is a linear combination of the three misalignments $m_{l}, m_{2}$ and $m 3$ according to:

$$
x=q^{3} a_{31} m_{1}+q^{2}\left(a_{21} m_{1}+a_{22} m_{2}\right)+q\left(a_{11} m_{1}+a_{12} m_{2}+a_{13^{m}}\right)
$$


where $a_{i j}$ are coefficient depending only on the optics of the system. Hence, given a sufficient number of " $x$ " measurements at different triplet strengths, it is possible to build an over determined linear system in terms of the unknown misalignments. The situation in slighuly more complex if we want to take into account the incoming beam centroid jitter to improve the accuracy of the method, therefore the algorithm has been generalized to fit for these additional unknowns.

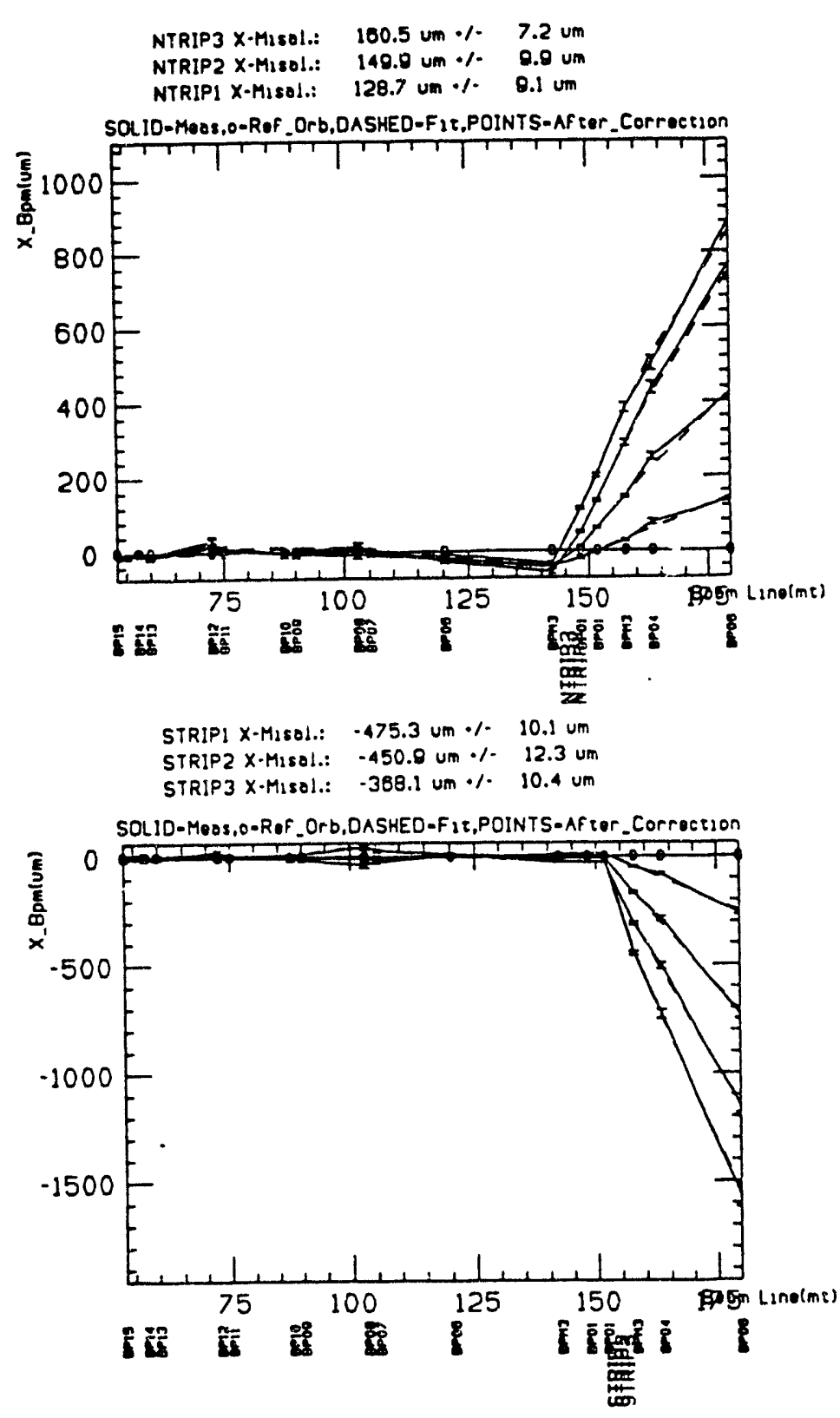

Figure 3. Fit of the $\mathrm{X}$-beam-orbits at different triplet strengths for the north and south triplets with respect to the same reference orbit. The increased steering for increased strengths is clear.

\section{RESULTS}

In fig. 3 is shown a typical output from the data analysis and table 1 summarizes the evaluated triplet misalignments before and after a move showing residual misalignments of the order of $30 \mu \mathrm{m}$ (1992 data). The misalignments are expressed in terms of average offset and tilt of the whole triplets since is possible to move them as a whole unit (see fig.1).

Table 1

Measured triplet misalignments before and after movement.

\begin{tabular}{|l|c|c|c|c|}
\cline { 2 - 5 } \multicolumn{1}{c|}{} & \multicolumn{2}{c|}{ North Triplet } & \multicolumn{2}{c|}{ South Triplet } \\
\cline { 2 - 5 } \multicolumn{1}{c|}{} & before & after & before & after \\
\hline$X \_$offset $(\mu \mathrm{m})$ & -357.4 & 3.0 & 303.7 & -44.0 \\
\hline$X^{\prime}$ offset $(\mu \mathrm{rad})$ & -78.0 & 0.7 & -66.2 & 9.7 \\
\hline$\left.Y_{\text {_offset }(\mu \mathrm{m})}\right)$ & 93.8 & 29.3 & 10.5 & -13.5 \\
\hline$Y^{\prime}$ _offset $(\mu \mathrm{rad})$ & 20.5 & 6.4 & -2.3 & 2.9 \\
\hline
\end{tabular}

The overall steering turning then on/off due to the residual triplets misalignments after correction is typically of the order of $30 \mu \mathrm{rad}$, causing orbit distortions easily compensated with correctors with negligible contribution to the IP-dispersion and to detector backgrounds.

\section{CONCLUSIONS}

The procedure has been successfully used in the SLDSLC 1992 and 1993 runs. In this last run in particular, since an upgrade in the BPM resolutions in the Final Focus, it has been possible to reach a resolution better than $10 \mu \mathrm{m}$. With this improvement, the electrons and positrons beams are steered so little from the reference orbit that they are usually colliding immediately after the triplet alignment. 

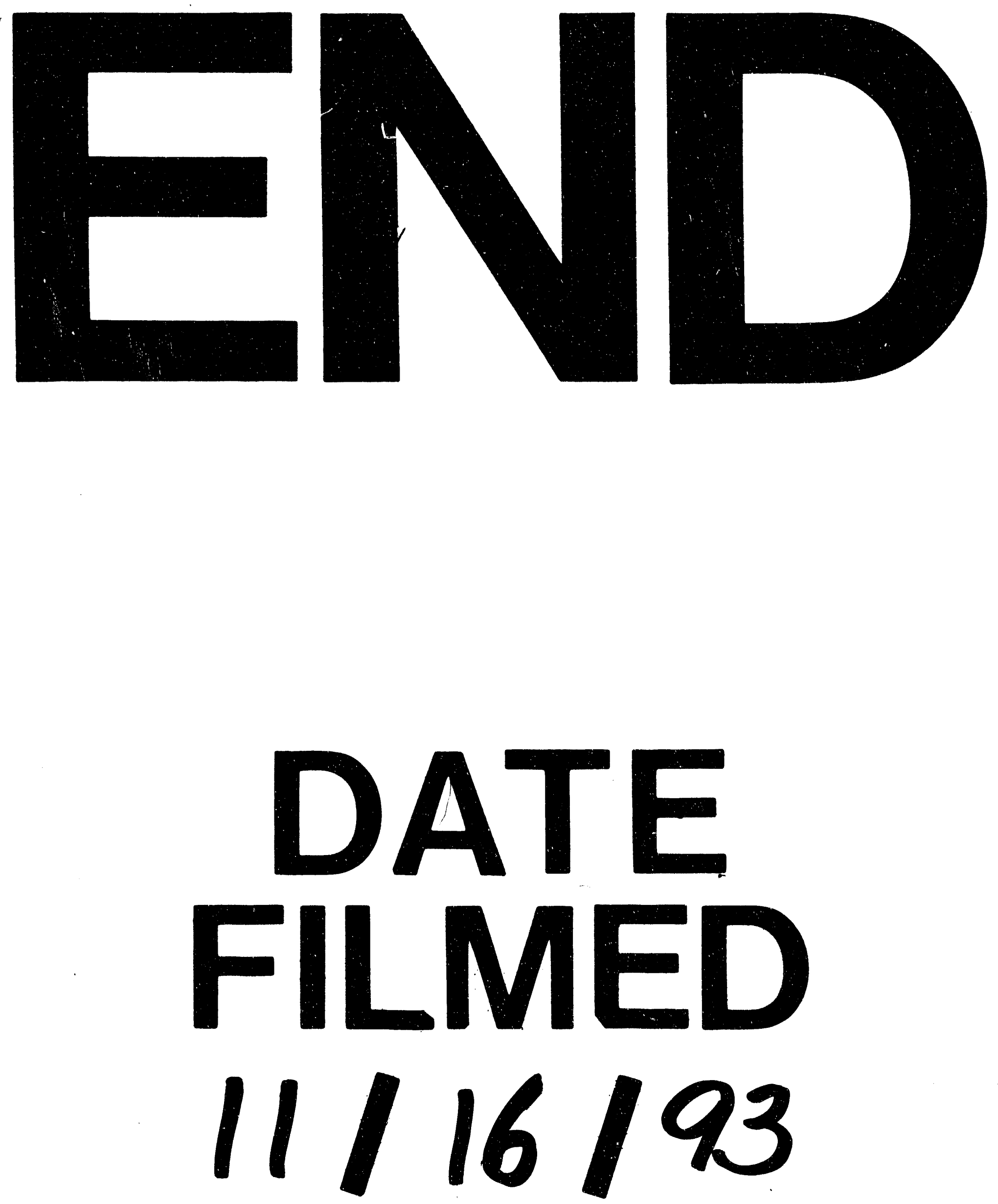
